\title{
KEMBS
}

\section{premier échelon \\ du Grand Canal d'Alsace}

De nombreuses études ont paru sur l'usine de Kembs. Cependant, il nous a semblé que it Houille Blanche, se devail de documenter ses lecleurs sur les particularités d'ordre hydraulique d? ses aménagements. L'Energie Electrique du Rhin nous a aimablement autorisé à extraire de l'oubrage rcmarquable qu'elle a fait paraître sur l'Usine Hydroélectrique de Kémbs (1), les quelques pages qu' vont suive et nous a fourni les photographies qui les illustrent. Nous tenons à leur adresser ici nos plus vifs remerciements.

\section{Le Rhin et son aménagement}

Le Rhin, à sa sortie de Suisse, est alimenté par un bassin de $35.930 \mathrm{~km}^{2}$, dont $700 \mathrm{~km}^{2}$ de glaciers et $1.000 \mathrm{~km}^{2}$ de lacs. Son débit est partiellement régularisé par les lacs et par les tement en aval de Bâle de $1,1 \%$ à Strasbourg de $0,65 \%$ et à Mannheim de $0,20 \%$.

Au commencement du siècle passé, le cours du Rhin dans la plaine d'Alsace présentait encore de nonbreux méandres et sé

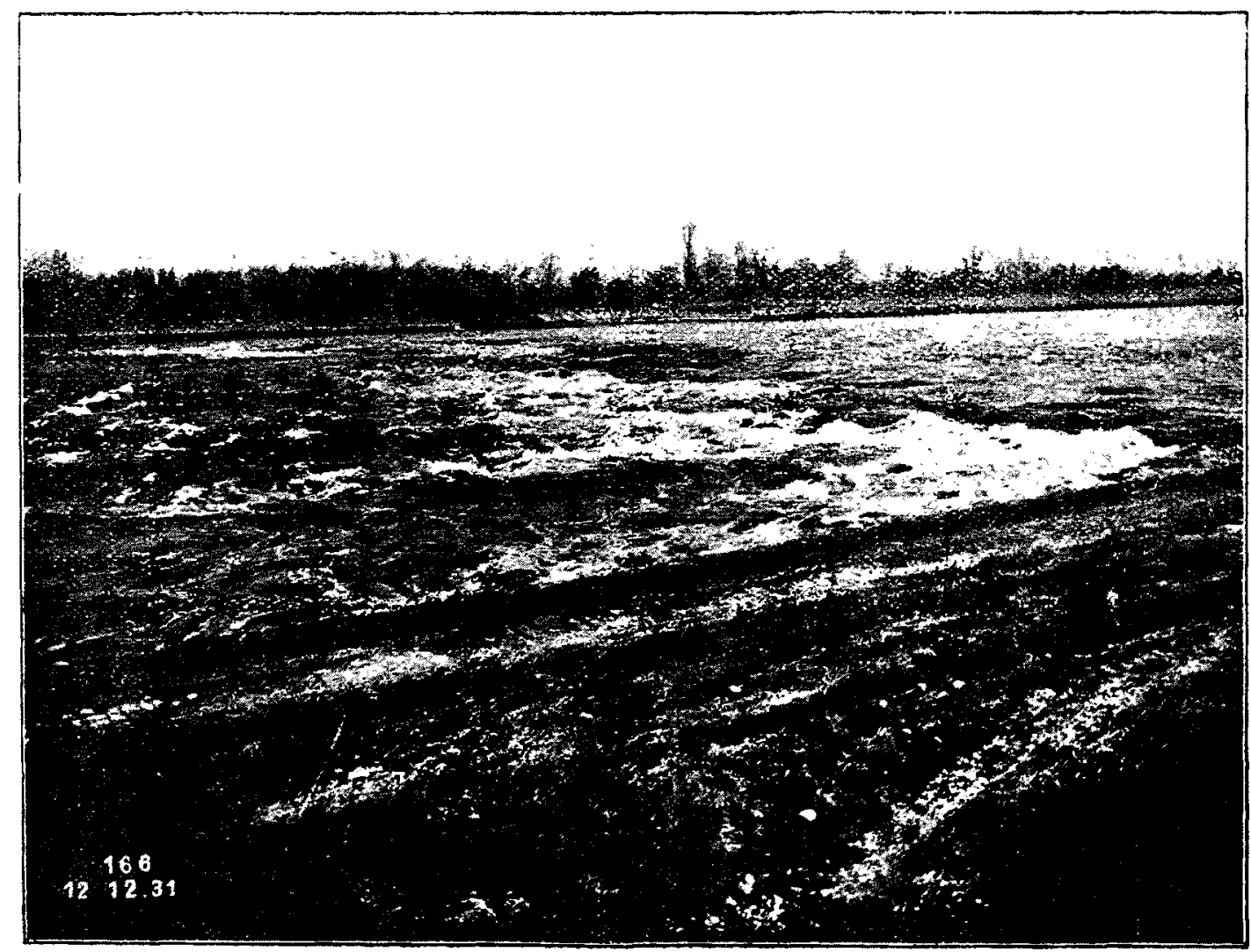

Fig 1.- La barre d'Istein_le 12 décembre 1931

glaciers ; mais il présente cependant encore les variations suivantes :

Débit semi permanent (réalisé 6 mois par an). . . $1.000^{\mathrm{m} 3} / \mathrm{sec}$. Débit d'étiage ................... $380 \quad \ldots$ Crues ordinaires (en moyenne 3 jours par an env.), 2.400 Crue extraordinaire maximum $(1876) \ldots \ldots \ldots .6 .000$ -

Le Rhin, comme tous les fleuves, présente un profil en long de forme parabolique. De l'amont à l'aval, son débit augmente et le grain des alluvions diminue en mème tunups que la pente. modifiait d'année en année. Pour protéger les riverains, on aval bien construit les digues des hautes eaux, mais celles-ci étaiell souvent détruites par les crues exlraordinaires et le fleuve cauball alors de véritables dévastations.

Pour porter remède à cet état de choses, le Gouvernement Français, en 1840, se mit d'accord avoc le Gouvernement Bador pour entreprendre une correction rationnelle sur tout son pat cours.

On a ainsi formé, à l'aids de deux digues, parallèles, un ll 
mineur d'une largeur de 180 à 250 mètres dans lequel coulent les eaux en temps normal, tandis que le leuve s'étale au delà de ses digues en cas de crue extraordinaise et forme un lit majeur, linité par les digues des hautes eaux situees à droite el à gauche du lit mineur.

La correction du Rhin a enlraîné un raccourcisscment sensible du lit du fleuve, dont la longueur entre Bâle ct Mannhei.n s'est trouvée réduite de 354 à 275 kilomètres, soit de $23 \%$ (le long de la frontière française celte réduction est de $14 \%$ ).

Par suite dı raccourcissement provenant de la correction, la pente par kilomètre du fleuve a été augmentée et l'équilibre du fleuve a été rompu : le Rhin se trouve avoir un excédent de force vive qu'il emploic à creuser son lit, et à se donner un nouveau profil en long d'équilibre, dont la pente est plus faible que la pente actuelle. Il s'en suit un approfondissement du lit allant enl augmentant de l'aval à l'amont. C'est ainsi qu'à Kembs, un peu on amont de Mulhouse, le Rhin s'est creusé depuis la correction d'environ 4 mètres, tandis qu'à l'aval de Brisach l'approfondissement est encore nul.

Linfluence de cette érosion remontante est toutefois netlement déli nitée par la barre rocheuse d'Istein, située à environ 9 kilomètres en aval de la frontière suisse ct à 5 kilomètres en amont de Kembs. Dạns unt conférence que M. René Koechlin a faite à la Société Industriclle de Mulhouse en 1906 , il avait déjà signalé l'existence de cette barre rocheuse, dont on pouvait constater la présence au fond du lit du Rhin, et il prévoyatt que, par sute de l'approfondiss mment graduel de celui-ci, il se formerait en cet endroit des rapides qui deviendraient plus tard un obstacle à la navigation.

A ce moment là, le banc de calcaire était encore en moyenne au-dessous du lit du Rhin et sa présénce passait presque inaperçue. On remarquait seulement, à cet endroit, un léger accroissement de la vitesse du courant. Aujourd'hui, après 26 ans, les rapides sont déjà considérables comme on peut le voir par la fig. 1.

La barre d'Istein se compose de deux bancs de calcaire parallèles et distants de 400 mètres environ, qui traversent le lit du fleuve. La différence entre le plan d'eau-au-dessus de la barre et celui en aval du banc de rocher inférieur est d'environ 2 mètres. La vitesse du courant à' la barre d'Istein est d: 4 à 5 mètres à la secondé.

Si la navigation en aval de Stràsbourg đañs le Rhin régularisé est déjà très difficile, elle l'est incomparablement davantage entre Strasbourg et Bâle. Sans parler de la barre d'Istein, qui constilue déjà un obstacle très sérieux à la navigation, il est clair qu'avec les vitesses de courant de 3 à 4 miètres par seconde que l'on rencontri en aval de Bâle, la navigation ne peul plus être économique. Même en employant des remorqueurs de 800 à $1.000 \mathrm{CV}$, on ne peut remorquer jusqu'à Bâlc que duux chalands chargés au maximum de 500 tonnes chacun, alors que les mêmes remorqueurs amènent jusqu'à Strasbourg deux chalands chargés de -1.000 à 1.200 tonnes chacun, ei, jusqu'à Mannheim, deux chalands de 2.500 tonnes chacum. Pour passer la barre d'Istein, le remorqueur montait d'abord seul, s'amarrait et tirait ensuite chaque chaland séparément à l'aide de cabestans, opération très délicate et très dangereuse (1).

Si l'on ajoute à tous ces inconvénients que le Rhin, entre

(1) Il est hors de doute que sans la dérivation, aujourd'hui effectuée, du canal de Kembs, qui permet aux bateaux de contourner la barre distein, celle-ci aurait, en peu d'années, rendu toute navigation impossible.
Strasbourg et Bâle, n'est navigable ni en périodes de basses eaux, ni en périodes de haules caux, et que le service ne peut donc se faire qu'à certaines époques réparties inégalement dans l'année, en tout pendant 100 à 200 jours, on voit combien cette navigation sur le Rhin libre est précaire et peu économique, et combien la régularisation du Rhin entre Kembs et Bâle serait un mode d'aménagement incomplet mème pour la navigation.

L’aménagement du Rhin, en amont de Bâle, s'est fait par chutes successives (Rheinfelden, Augst-Wyhlen, Laufenbourg, Eglisau, Rybourg-Schworstadt, Dogern), uniquement pour l'utilisation de la force motrice, sans tenir compte des besoins de la grande navigation.

Pour la partie du Rhin en aval de Strasbourg, au contraire, on a régularisé le Rhin en enlevant toute possibilité d'utiliser sa force motrice.

L'idée d'aménager le Rhin entre Strasbourg ct Bâle n'est venue que plus tard. A ce moment-là, il n'était plus possible, comme le voulaient les partisans du Rhin libre, d'envisager le problème uniquement au point $d t$ vue de la navigation, sans

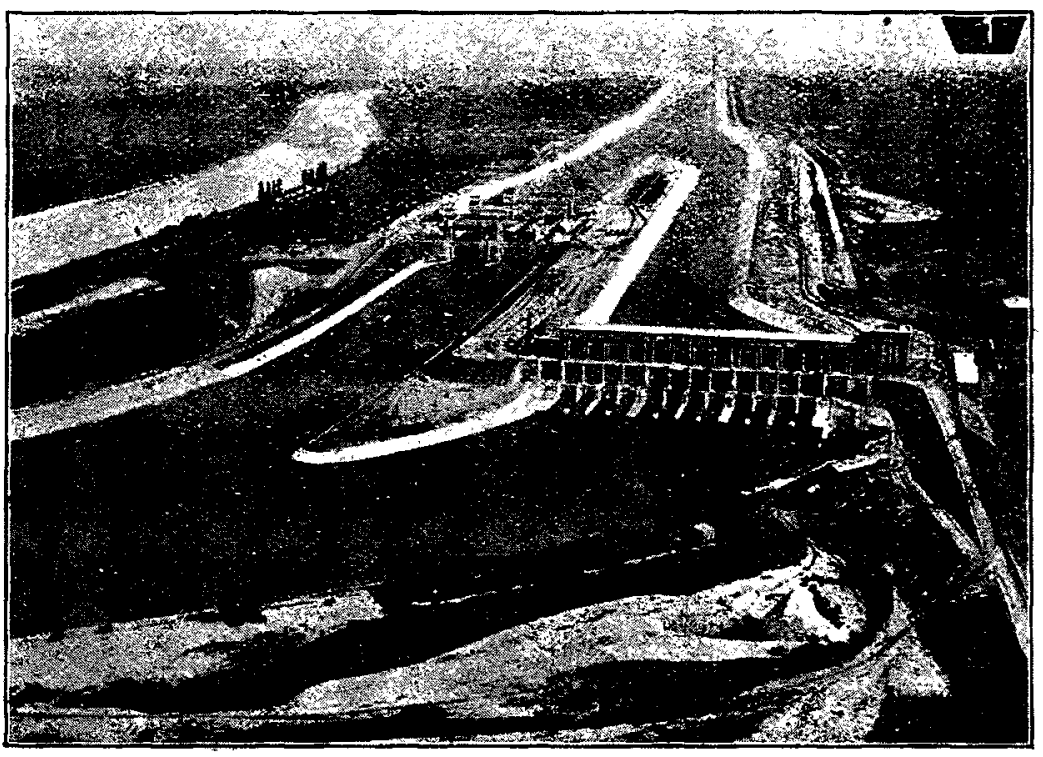

Fig. 2.-- Vùe générale des aménagements de Kembs. Au premier plan, a gauche, le canal de fuite. Au centre, le canal de navigation avec les écluses, la centrale et le canal d'alimentation des turbines. En arrière, le canal d'amenée. Au second plan, le Rhin.

tenir compte de l'utilisation de la force motrice. La puissance réalisable sur le Rhin entre Bâle et Strasbourg, est, en effet, considérable.

La chute disponible sur ce parcours est de 108 mètres. Pour un débit moyen de $850 \mathrm{~m}^{3}$ par seconde, la puissance réalisable est en aval de Bâle de $9.300 \mathrm{CV}$ par kilomètre de fleuve. A Strasbourg, elle est encore de $5.500 \mathrm{CV}$. La puissance disponible entre Bâle et Strasbourg est donc en moyenne d'environ $7.300 \mathrm{CV}$ par kilomètre; en hasses eaux, elle est encore de $5.800 \mathrm{CV}$. par kilomètre.

L'idée d'utiliser la force motrice du Rhin entre Bàle et Strasbourg avait déjà été émise, en 1837, par M. Fourneyron, en collaboration avec M. Emile Koechlin. Plusieurs projets d'aménagement ont élé présentés avant la guerre; mais ils n'ont pu aboulir, car iis étaient entravés par des groupements ayant des intérèls contraires.

C'cst le 9 juillet 1919 que M. René Koechlin et la Société des Forces Motrices du Haut-Rhin, formée le 1er août 1910 à Mulhouse, déposèrent lo projet du canal latéral au Rhin, appelé 
ensuite Grand Canal d'Alsace. Ce projet fut combattu tout d'abord par les partisans du Rhin libre, mais fut finalement accepté à l'unanimité par la Commission Centrale pour la Navigation du lihin, par ses résolutions du 16 décembre 1921 el 10 mai 1922, pour l'échelon de Kembs, et par sa résolution du 29 avril 1925 pour le reste du Grand Canal d'Alsace.

Pour l'exploilation ct la mise en valeur de ces concessions, la Société des Forces Molrices du Haut-Rhin et M. René Koechlin ont fondé la Société Energie Electrique du Rhin, qui a été définitivement constituée le 10 décembre 1927 et substituée aux Forces Motrices du Haut-Rhin,.

En établissant l'avant-projet général du Grand-Canal d'Alsace, M. René Koechlin était parti des principes suivants :

10 La chute totale disponible entre l'extrémité du remous (frontière suisse) et Strasbourg devait être partagée, autant que possible, en chutes à peu près égales.

\section{Description des installations}

Le canal de Kembs, qui constitue la première des huil sections du Grand Canal d'Alsace, s'embranche sur le lhin au kilomètre 5 de la rive française, c'est-à-dire à 5 kilometres on aval de la frontière suisse. Un grand barrage mobile esl ilabli au kilomètre 5,5, où il relòve le niveau du Fhin d'environ 7 mètres en basses eaux, le seuil du barrage étanl élabli all niveau moyen du lit du Rhin. En temps de grande "crue, les vannes du barrage sont complètement levées el les conditions d'écoulement du fleuve restent les mêmes qu'auparavant.

Le relèvement du plan d'eau au barrage a été calculć de manière que le remous en amont s'efface complètement au débouché de la Birse dans le Rhin.

Le canal d'amenée sert à la navigation el à l'alimentation des turbines; avant d'arriver à l'usine, il se sépare en deux

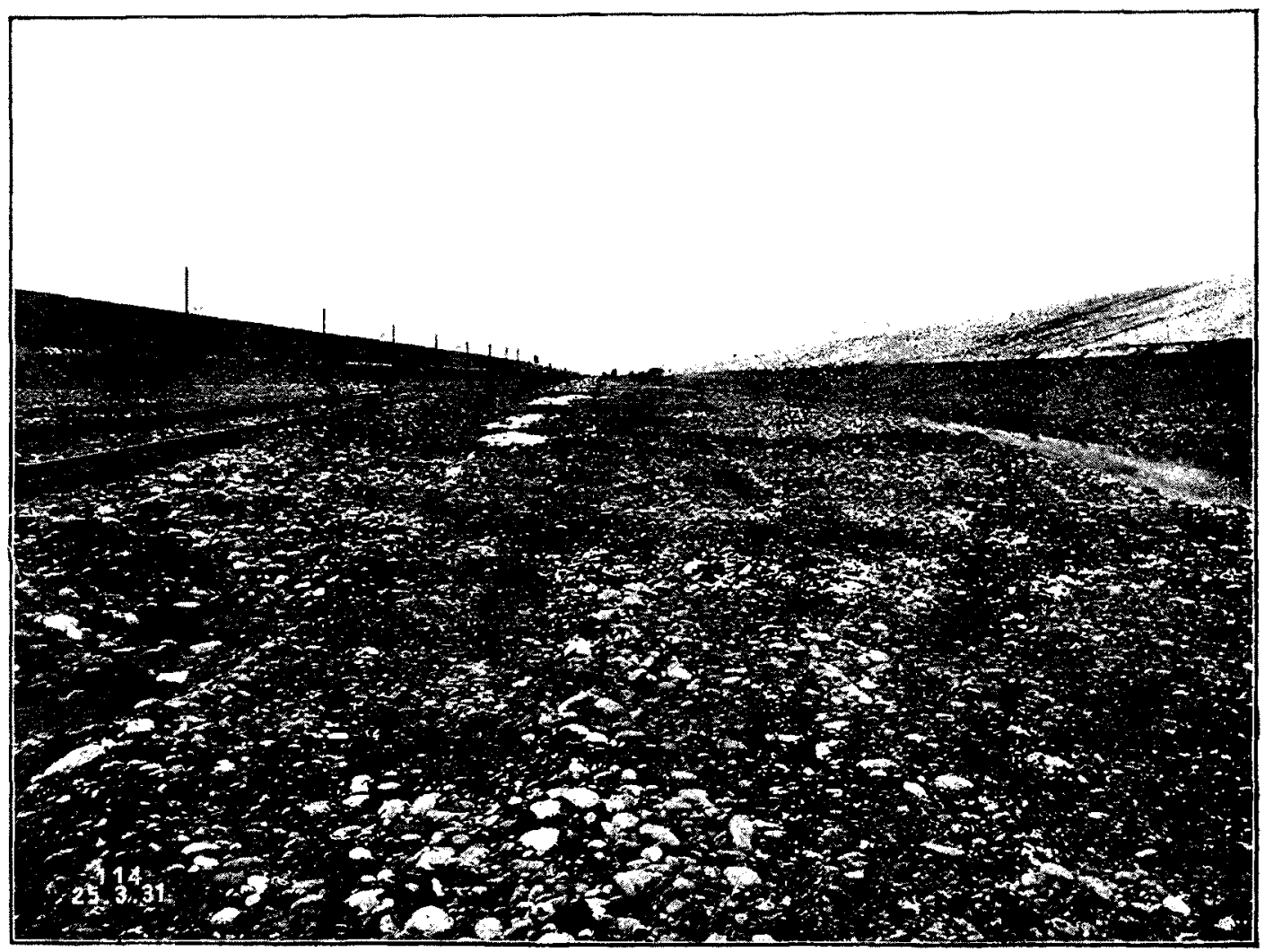

Fig. 3. - Profil du canal terminé

$2^{\circ}$ La différence de niveau entre le plan d'eau maximum dans les canaux et le sol ne devait en aucun point être supérieure à 8 à 9 mètres ; le plafond du canal devait ainsi toujours se trouver dans le terrain naturel.

$3^{\circ}$ Les battiments des turbines devaient être situés, autant que possible, près du Rhin, de manière à raccourcir les canaux de fuite provisoires rendant l'eau au Rhin.

Ces principes, qui ont conduil à partager la chute totale entre Bâle et Strasbourg en 8 échelons, ont èté conservés dans le projet détaillé du Grand Canal d'Alsace, établi par le Consorlium des Canaux d'Alsace-Lorraine (1). Les rhutes successives, un aral d. Kentus, rari thl enir: 11 of 1.1 metres, suivant la disporition d's li ux.

(1) Les usines prevues entre Bàle et Strasbourg sont : Kembs, Ottmarsheim. Fessenheim, Vogelgrun, Marckolsheim, Sundhouse, (icistheim, Strasbourg. branches, l'une réservée exclusivement à la navigation, l'aulté exclusivement à l'amenée d'eau aux turbines (fig. 2).

Du côlé du canal de fuite, le canal d'amenéc est fermé par l'usine-barrage et le canal de navigalion par les écluscs. En aral de la chute ainsi formée, les biefs inférieurs des écluses el dis lurbines se rejoignent el le canal de fuite, qui est très coutt, ramène les eaux dans le Rhin au kilomètre 11,8 , en allendant qu'il soit prolongé pour desservir la seconde chule.

Le Grand Canal d'Alsace doit être considéré comme une dér: vation du Rhin débitanl normalement $850 \mathrm{~m}^{3}$ par seconde tandis que le reste du débit du fleuve s'écoule par le lil du Rhin Pour maintenir ca débil dans le canal, en vue des chutes aval, mème quand les Lurbines de Kembs seraient partiellement arrêtées, un déversoir corruspondant a été aménagé à travơs l'usine.

C'est l'usine-harrage de Kembs qui forme l'ouvrage de garde du Grand Canal d'Alsace. 
Barrage et Prise d'eau. - - I.e barrage, qui est établi perpendiculairement au Rhin, se compose de cinq ouvertures de 30 mètres séparées par des piliers de 5 metres dépaisseur at fermées chacune par deux vannes métalliqucs superposées, conslituées par des parois verlicales en tôie, garnies de poutros horizontales qui reportent la pression sur les piles ou les culéc.

l'écoulement des eaux s'uffectue soil en soulevant la vanne inféricure (système Sloney), -- ce qui permet cñ même temps d'évacuer les graviers -- soit en abaissant la vanne supéricure, ce qui permet de laisser passage à la glace cn hiver.

In ascenseur à poissons est disposé dans la culée gauche. Un filet du genre des filets à saumon monte et descend alternativement, fonctionnant comme un vérilable ascenseur. Un courant d'eat, destiné à attirer les poissons, traverse l'appareî. En outre, une échelle à poissons ordinaire cst établie sur la rive gauche. de mètres cubes de terrassements et $465.000 \mathrm{~m}^{3}$ de béton, étant donné, d'autre part, l'obligation d'exécuter ces travaux dans une période relativement courle, il a été nécessaire d'avoir recours à des procédés perfectionnés et à des engins spéciaux, construils souvent spécialement sur demande.

Les talus sont protegés et rendus étanches par un revêtement de beton, dont l'épaisseur varie suivant les cas de 12 à 20 centinctros dt qui contient un truilis ntaiique. Ce revetement part du couromement et descend jusquien dessous des eaux souterraincs. Il s'est effectué de la facon suivante:

'Tout d'abord, on a régle soigneusenent le lalus. Ce réglage s'ust fait à la main dans une partie du canal d'amenée, et dans l'autre partis à l'aide d'un apparéil conçu spécialement pour ce travail (fig. 4).

Il se compose d'une charponte métailique, embrassant tout le talus et se déplaçant sur des voies, situees l'une au pied de la digue, l'autre au couronnement de celleci.

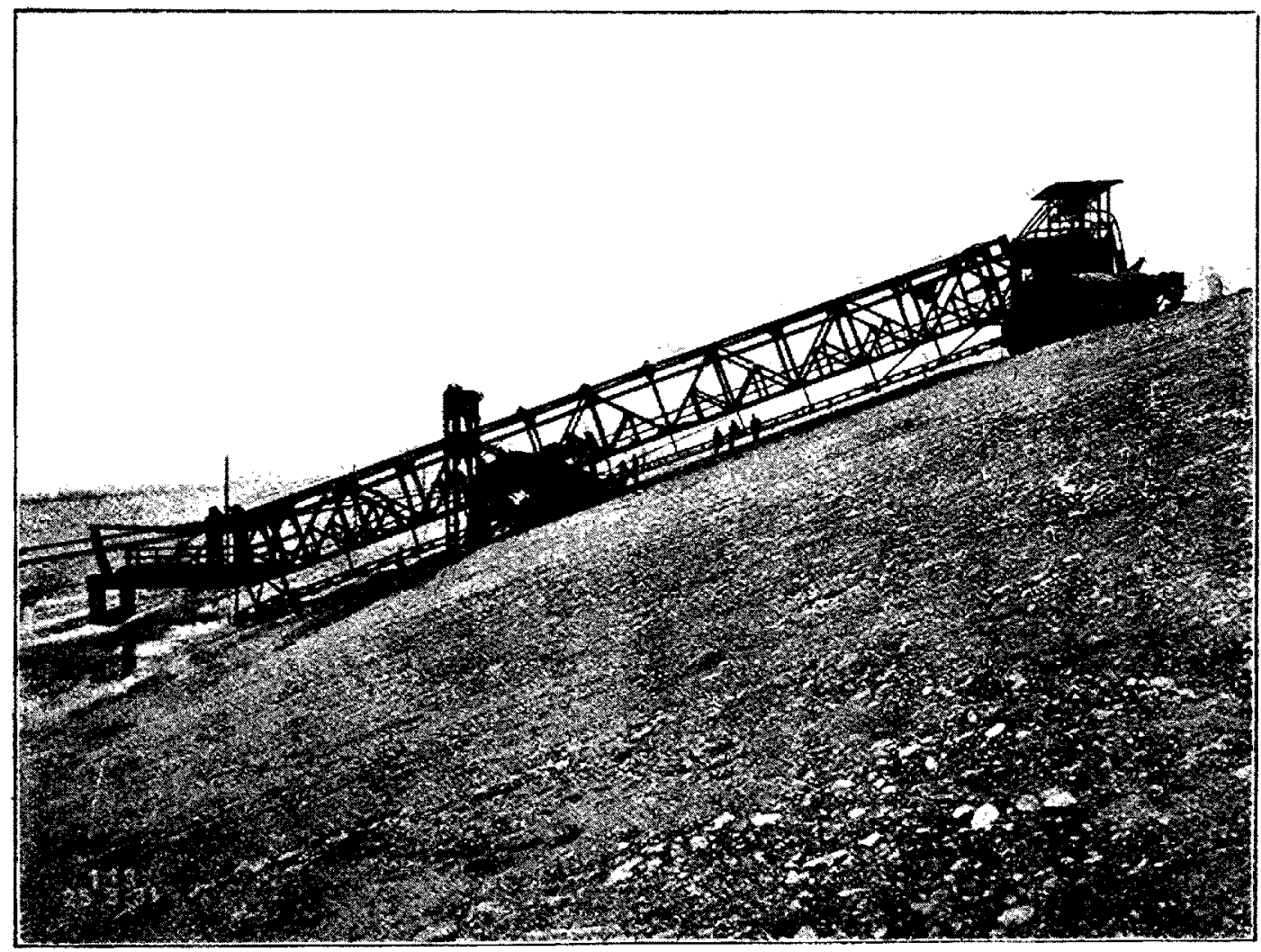

Fig. 4. - Les machines a régler les talus. Dans le fond on aperçoit le talus opposé

Canal d'amonée. - - Le canal d'amenée a une largeur de 80 mètres all plafond et d environ 150 mètres à la surface d'cau maximum. La profondeur d'eau esl d'environ 12 mètres (1) (fig. 3 .

I.a vitesse moyenne de l'eau par seconde ne dépasse pas $0 \mathrm{~m} .70$ pour un débit normal de 850 mètres cubes par seconde

Les digues du canal ont des talus de 3 à 1 et une largeur de couronnement de 15 mètres au minimum. Etant donné d'une part l'importance spéciale des travaux, qui comportaient en particulier, pour le canal, l'usine et les écluses, plus de 7 millions

(1) A titre de comparaison le canal de Suez à une largeur de 45 à 6i) mètres au plafond et de 10 l a $1 \geqslant 0$ mètres a la surface de l'eau. Sa profondeur est de $13 \mathrm{~m}$. La pente des digues est de $3: 1$, comme celle du Grand Canal d'Alsace. Le canal de Panama a une largeur de $41 \mathrm{~m} .50$ au plafond La pente des digues étant de 1: 10, la lar. geur au niveau de l'eau est sensiblement la mème. Sa profondeur est de 13 mètres. Le Giand Canal d'Alsace est donc nettement plus important que le canal de Suez et le canal de Panama.
Sur ce pont roulant sont disposés différents appareils permettant d'enlever les remblais aux endroits ou ils sont en excédent, d'en apporter où il en manque et de pilonner ensuite tout le talus pour assurer au revêtement une bonne surface d'assise.

Le bétonnage des talus s'est effectué ensuite à l'aide de deux appareils spéciaux (fig. 5) dont la charpente mètallique était analogue a celle de l'appareil servant au réglage du talus. La aise en place du béton et du treillis métallique, ainsi que le pilonnage du béton, sc faisait mécaniquement à l'aide d'un chariot se mouvant le long de la charpente inclinée.

Chaque appareil pouvait mettre en place par heure environ $25 \mathrm{~m}^{3}$ de béton. Le lissage de la surface du béton s'est fait à l'aide de petits apparcils rotatifs, actionnés électriquement, que lon déplatait à la main el dont la construction est analogue a celle dis cireuses électriques.

Dans toute la partic aval du canal d'amenée, à partir de la bifurcation du canal des écluses, le plafond du canal a été revêtu 
d'une couche de béton de $0 \mathrm{~m} .20$ à $0 \mathrm{~m}$. 30, de manic̀re à éviter que l'cau ne passe par infiltration du bief amont an bief aval. de béton recouverte d'une couche proledries de gravier de

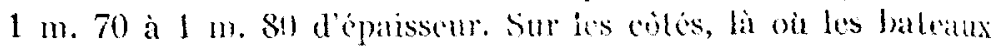

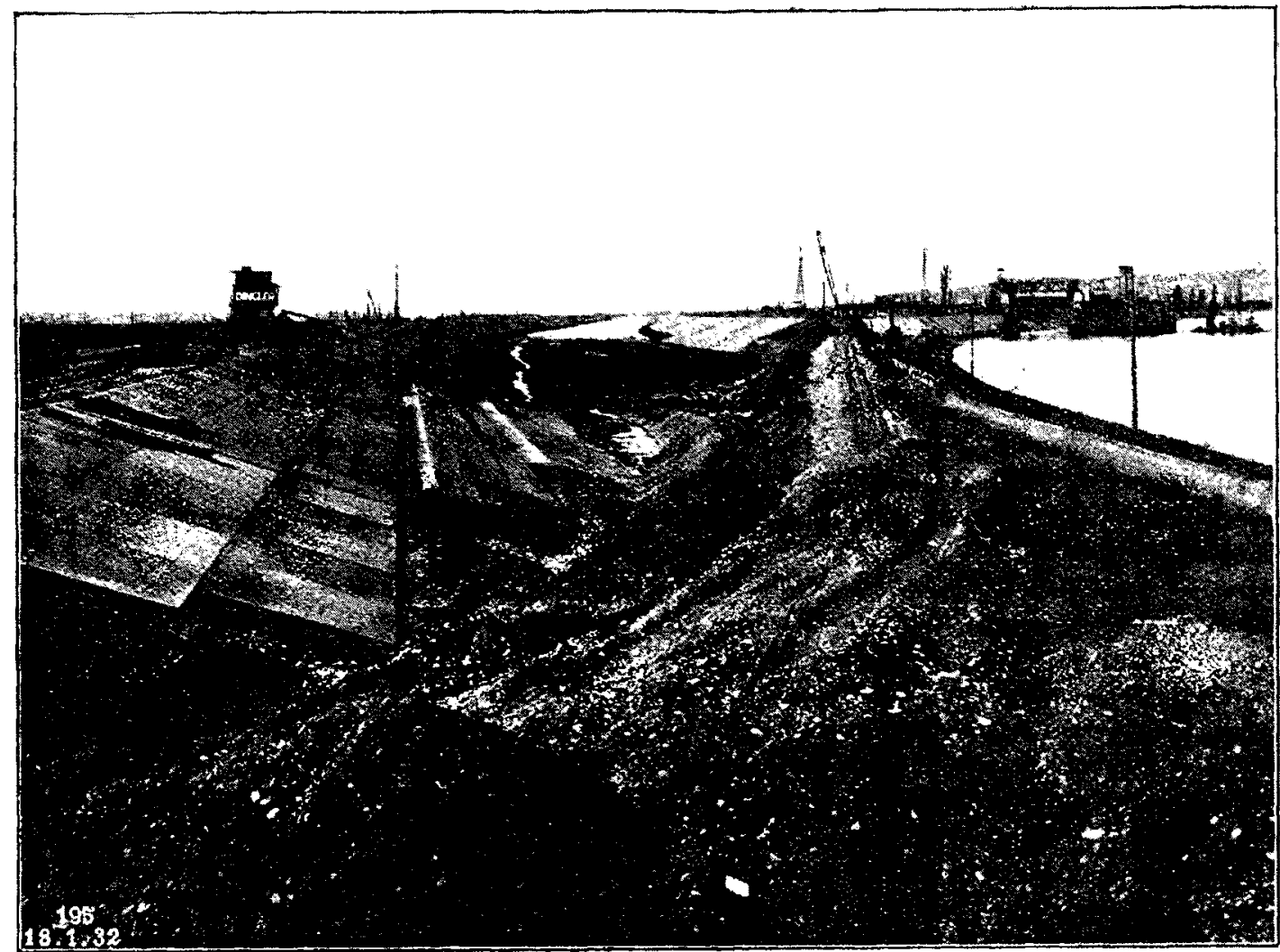

Fig. :... La bétonneuse des talus On voit la digue-bouchon séparant encore le canal d'amenée du Hhin (à droite), donl le nuesu est de 3 mètres au-dessus du plafond du canal Les eaux souterraines commencent à envahir te canal.

E-luses. ...- La dérivation du canal d'amenée allant vers les eclus s, a uno profondeur de $7 \mathrm{~m}$. 50 . Vers l'écluse, le plafond peuvent accoster, les talus gamis de béton avee latilis sont recouverts de pavages en pierre.

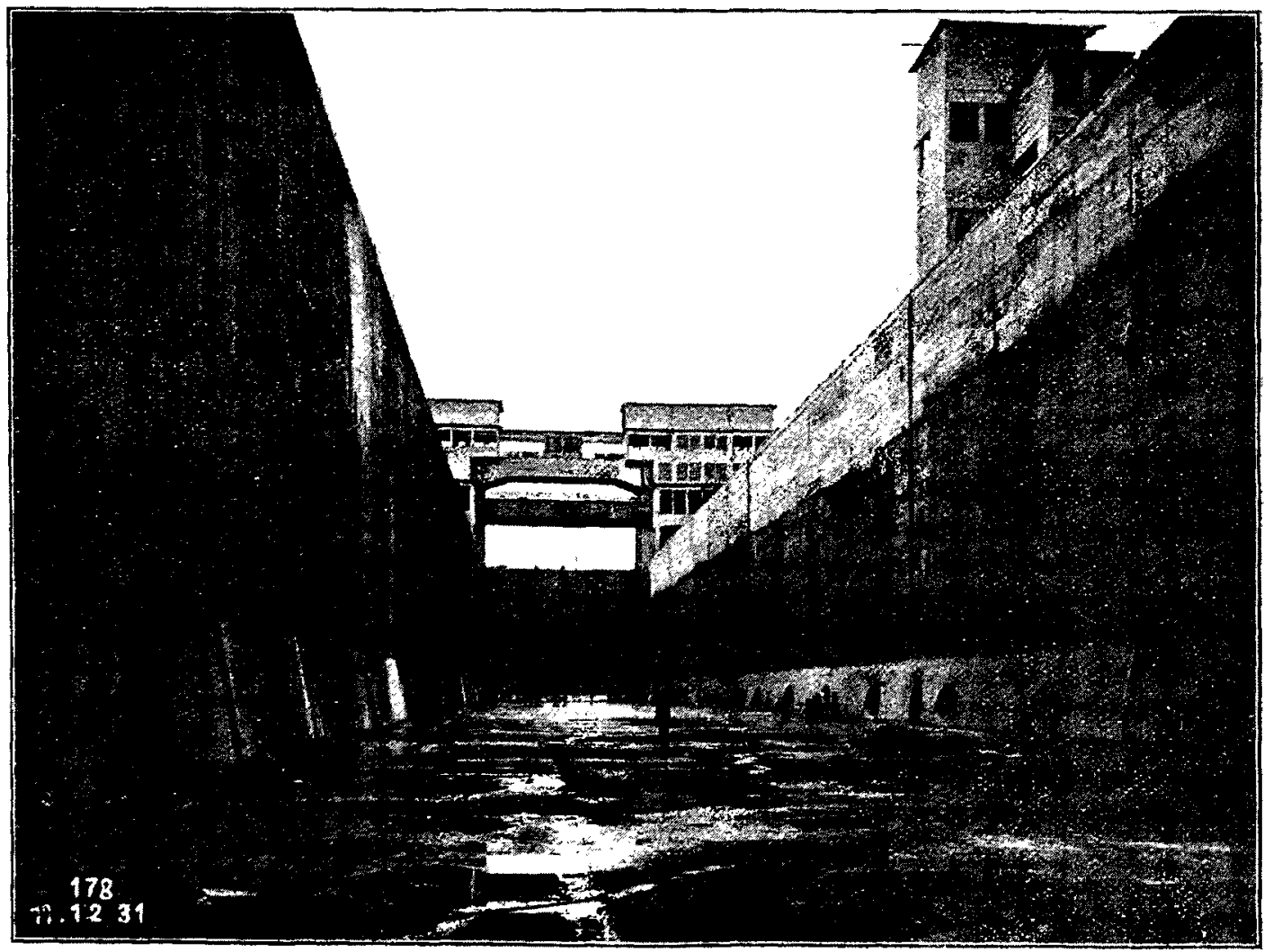

Fig. 6. - Vue du sas de la grande écluse

s'élargit sur une longueur de 185 mèlre's pour former un garage pour les bateaux. Le radier du canal esl constilué par une paroi
Les sas des deux écluses ont chacun une largen ulile do 25 mètres ; l'un a 185 mèlres de longucur, l'autre 100 nietrotst 
Ih sunt conslityes par des murs en beton is paross verticales de $20 \mathrm{~m} .50$ de hanteur a l'interieur et avec gradins a l'exterieur (fig. 6).

les portes d'icluses sont constimes par de grandes rammes en loles gann: dr pouldes horizontales. Biles conitissent vertacalement dans des faimures monagess dans la maconalric. la vanne aval de chaque écluse at ume hatuleur de $19 \mathrm{~m}$. 50 el son lablin seul pise plus de 500 tonnes ; la vanue amont a une hauteur de 8 mitr.s son lablire seul pèse environ 1011) lonnes, Les vannes aval sont equibluées par des contrepoids.

l'our remplir al vider les échuses, Jes hajoyers de celles-ci sont pourvus su loule leur longleur, à leur partue. inferi ure, douvertures metlant les sas cu communication avec des aqu dute nenagés dans les bajoyers, un ditus chique bajoyer de rive, deux dans l" bajoyer central. L'entrée de chaque aqueduc est commandé à lannonl et à l'aval des sas par des vamus. Si lon ouvre la vanne amont, lit vanm aval étant ferméc, le sas

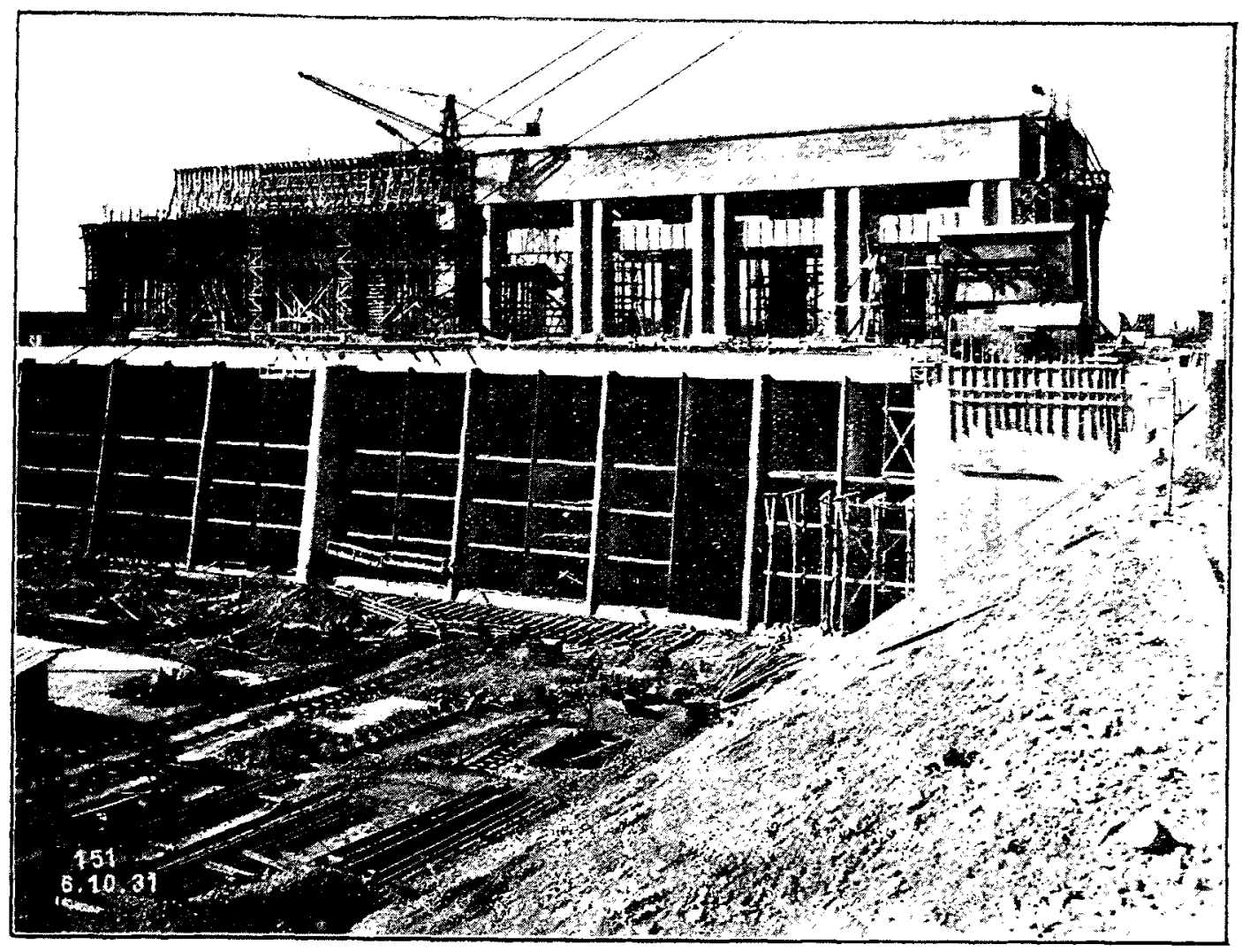

Fig. 7.- Vue amont de l'usine prise le 6 Octobre $193^{\prime}$. Dans la troisieme travée (4 travées;par turbine) la grille est poséc.

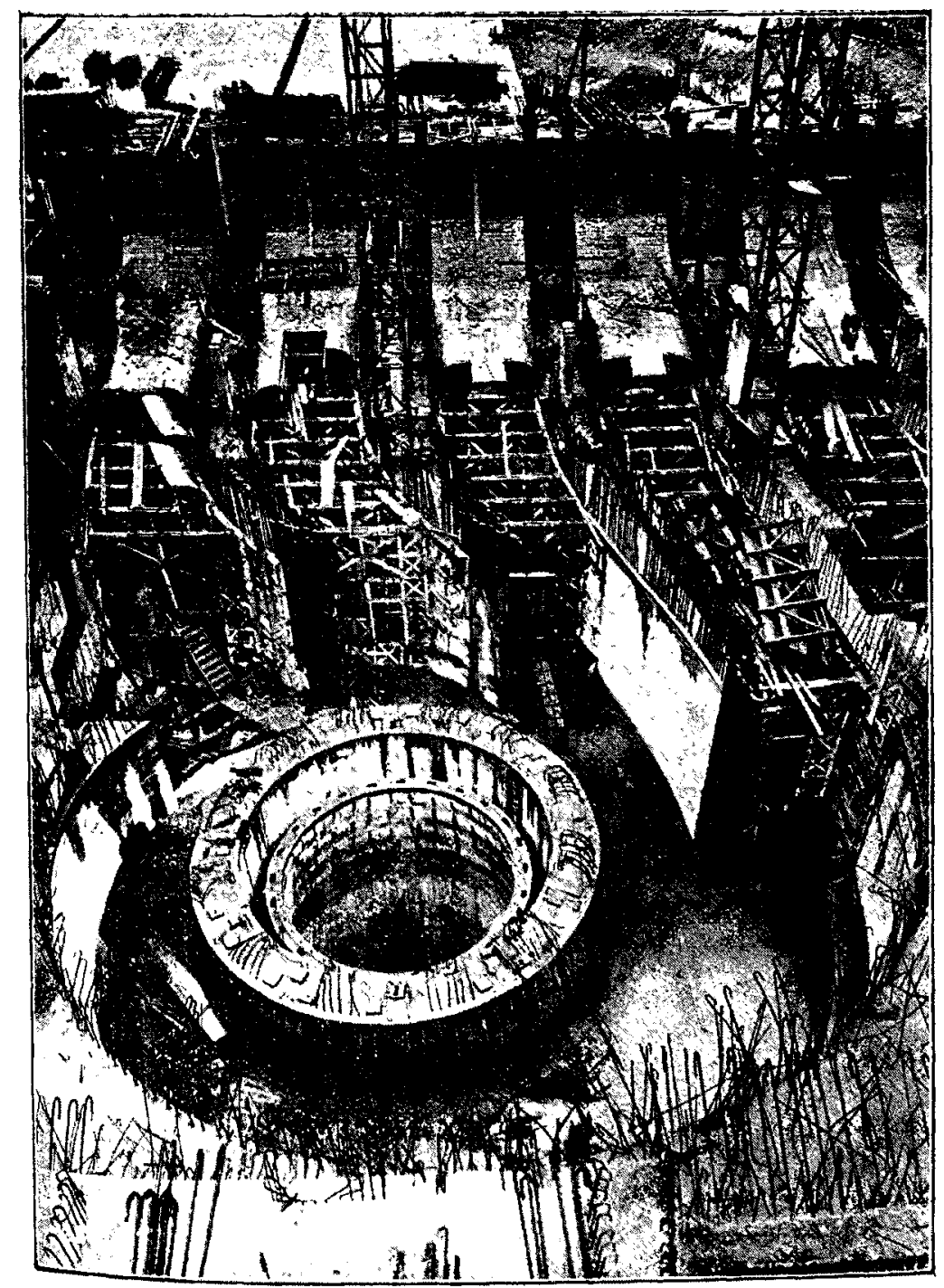

Fig. 8 - Cettc photographie, prise le 20 mai 1931, montre les 4 bâches d'entrée d'une turbine pendant leur bétonnage. Le distributeur de la de l'écluse se remplit; inversenent, si l'on ouvre la vamme aval, la vanne amont étant fermée, le sas se vide. Toules les manceuvres se font electriquement el permollent d'opérer l'éclusée d'un convoì de baleaux en moins d'une demiheure.

Usine. - L'usine de Kembs comporte 6 unites de turbo-allernaleurs à axe vertical dont une de réserve. Le bâliment dans lequel sont logés les lurbo-alterilateurs ot qui forme séparalion entre le canal d'amenéc el le canal de fuile, a une longueur entre-rives d'environ 130 metres; la largeur des maconneries entre la grille amont el le parafouille du radier aval est de 96 mètres (voir fig. 9).

Le bâtiment des lurbines proprement dit est divisé par des cloisons principales transversales en 6 seclions correspondant aux six groupes turbo-alternateurs (fig. 7).

Trois cloisons verticales transversales et une cloison horizontale partagent chaque section on 8 canaux; les 4 canaux inférieurs conduisent l'eau à la turbine apres son passage par la grille. Les cloišons de séparation des baches spirales se terminent par des-avant-hees en tôles (fig. 8).

Les quatre canaux supérieurs sonl fermés par deux vannes, la vanne du haul pouvant être abaissée pour le passage de la glace, ou relevéc, ainsi que la vanne infrieure, pour sorvir de déversoir. Le déversoir ainsi formé entre en action lorsque les lurbines sont totalement on partiellement arrêtées, de manière à maintenir constant le débit du canal. Ce dispositif très simple (1) a permis d'éviter l'établissement d'un déversoir spécial, qui aurait élé très coûteux (fig. 9).

1.es caux de chaque déversoir passent dans deux galeries à droite et à gauche de l'axe du groupe, qu'elles contaur-

(1) Disposilif breveté par M. René Koechlin et la Maison Locher et Cie. 
nent, et forment, à la sortie du bâtiment, une nappe se déversant audessus de la bâche d'évacuation des turbines (fig. 10).
Chaque turbine développe une puissance de $36.000 \mathrm{CV}$. sous une chute maximum de $16 \mathrm{~m} .60$ et avec un débit de $187,5 \mathrm{~m} / \mathrm{sec}_{\text {, }}$

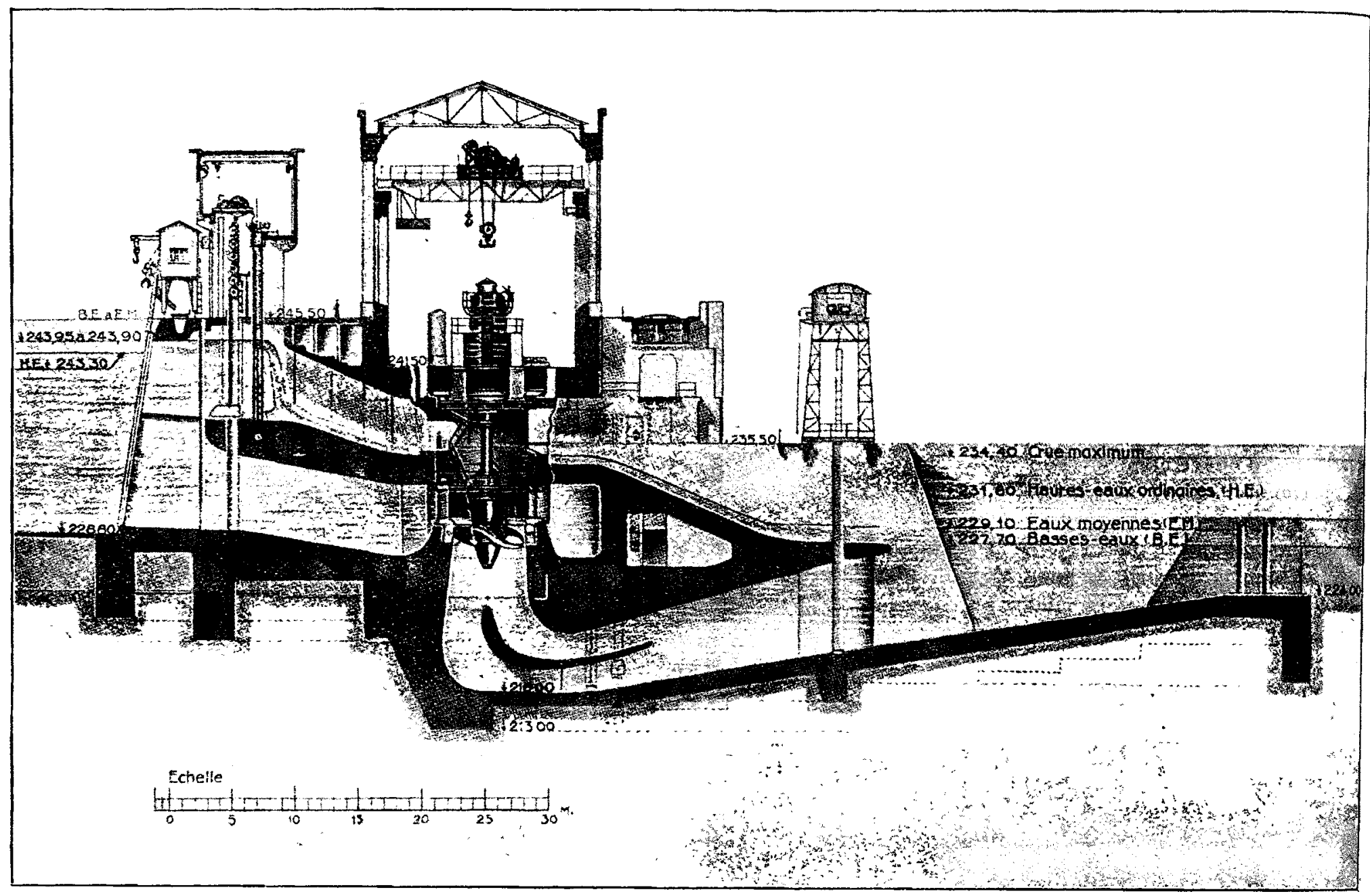

Fig. 9. - Coupe à travers le bâtiment des turbines

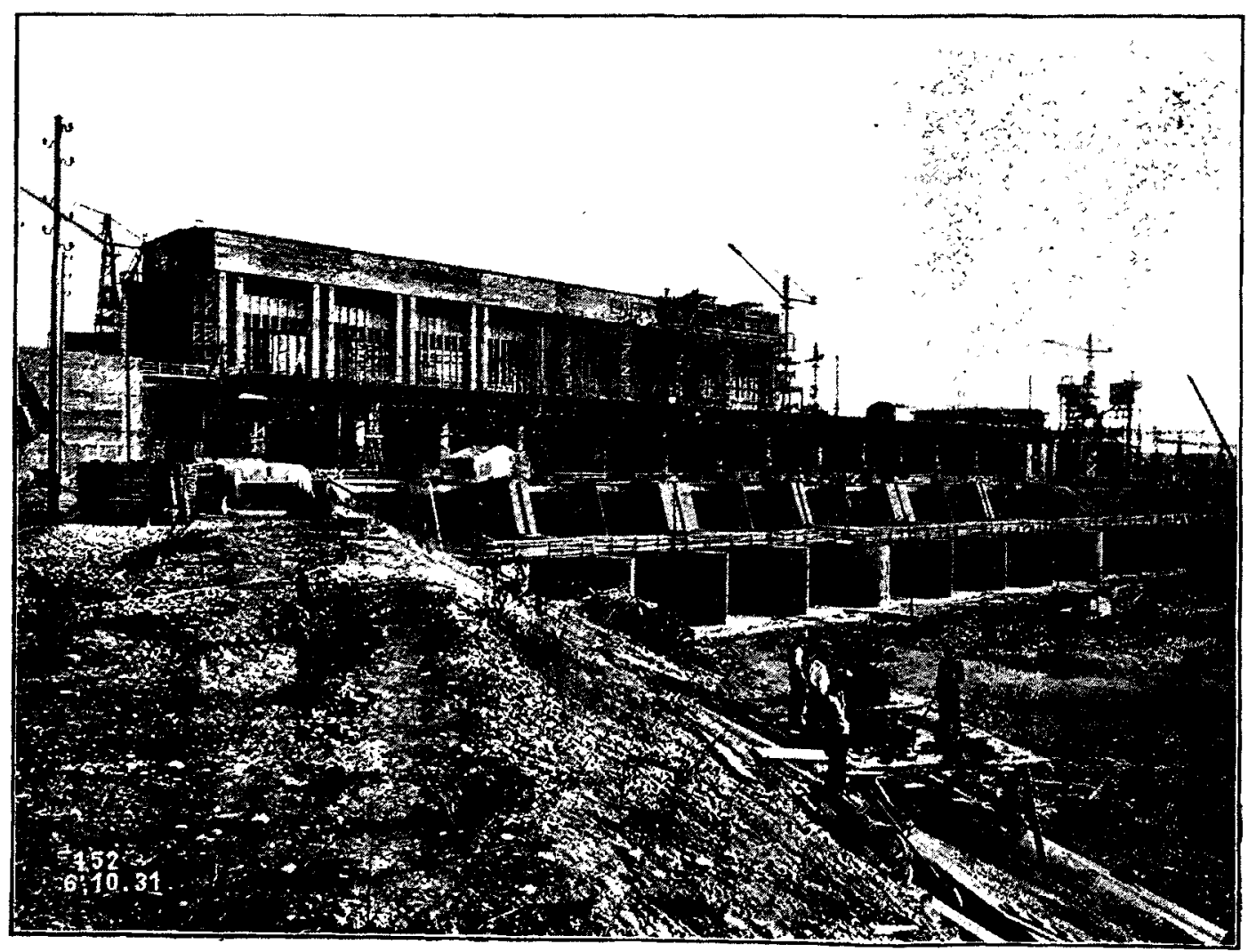

ce qui correspond pour les 6 turbines à une puissance installée d'environ $220.000 \mathrm{CV}$. La puissance da turbines diminue avec la chlut, de même que le volume d'eau absorbé par chaque turbine. Lorsque la chute nette est de 15 mètres, le Rhin permet l'utilisation d'un débit normal du $850 \mathrm{~m}^{3} /$ seconde et la puissance dis. ponible est alors de $150.000 \mathrm{CV}$. Dass une année d'eaux moyennes, l'usine de Kembs pourra fournir enviro 800.000 .000 kilowatt-heures.

L. Rateaux.

Fig 10.- Façade aval ale l'usine prise le 6 Octobre 1931. On distingue le pont de service qui portera la grue pour la mise en place des battardeaux. 